\title{
Magnetohydrodynamic Drop-on-Demand Liquid Metal Additive Manufacturing: System Overview and Modelling
}

\author{
V. Sukhotskiy ${ }^{1,2}$, P. Vishnoi ${ }^{3}$, I. H. Karampelas ${ }^{4}$, S. Vader ${ }^{2}$, Z. Vader $^{2}$, and E. P. Furlani ${ }^{1,3}$ \\ ${ }^{1}$ Department of Electrical Engineering, University at Buffalo SUNY \\ Buffalo, NY, USA \\ viktorsu@buffalo.edu; pvishnoi@buffalo.edu \\ ${ }^{2}$ Vader Systems \\ 385 Crosspoint Parkway, Suite 104, Getzville, NY, USA \\ ${ }^{3}$ Department of Chemical and Biological Engineering, University at Buffalo \\ Buffalo, NY, USA \\ ${ }^{4}$ Flow Science, Inc. \\ 683 Harkle Road, Suite A, Santa Fe, NM, USA
}

\begin{abstract}
We introduce an innovative additive manufacturing method that involves the drop-on-demand (DOD) printing of molten metal droplets to build three-dimensional (3D) metal structures of arbitrary shape. This technique is based on magnetohydrodynamic (MHD) droplet generation. Specifically, a transient magnetic field, generated by an electrically-pulsed external coil, induces a circulating current in molten aluminum that back couples to the applied field and creates a Lorentz force density (effective pressure) inside the printhead droplet ejection chamber. This effective pressure causes the ejection of a liquid metal droplet through a nozzle. Arbitrary 3D metal structures are printed in a layer-by-layer fashion. We present a commercial MHD-based printing system under development by Vader Systems (www.vadersystems.com) and introduce two computational models that predict system performance. We discuss the underlying physics of droplet generation and the thermo-fluidic aspects of droplet deposition, coalescence and solidification. We demonstrate good agreement between our computational models and measured data.
\end{abstract}

Keywords: Magnetohydrodynamics, Liquid Metal 3D Printing, Drop-On-Demand 3D Printing, Thermo-Fluidic Modeling, Liquid Metal Coalescence, Liquid Metal Solidification.

\section{Introduction}

Additive manufacturing (AM), often referred to as 3D printing, involves the fabrication of 3D structures (e.g. plastic) based on a digital CAD model, typically in a sequential layer-by-layer fashion. This emerging technology is in stark contrast to the traditional casting, forming, or subtractive manufacturing techniques in that it allows flexible, quick and inexpensive production [1]. In its early days, 3D printing was mainly used to build proof-of-concept prototypes and/or one of a kind objects. However, it has rapidly evolved towards the production of end-user products [2]. An area of particular and intense interest is AM of metal objects. Conventional metal AM techniques make use of lasers or electron-beams (EBs) as directed energy sources to fuse together specially prepared metal powder. Laser-based AM techniques include Direct Laser Fabrication Technique (DLF) [3], Selective Laser Melting (SLM) [4], and Laser Solid Forming (LSF) [5]. The EB techniques include EB Solid Fabrication (EBSF) [6], in which an EB is used to selectively melt the layer of metal in its powdered form. Similar to molten metal printing, the techniques also build components in a layer-by-layer fashion. However, these processes have drawbacks including both production costs and complex process control, which are due to their energy-intensive equipment needed to convert metal into powder form prior to fabrication, and the precise melting and fusing of the powder to form a desired 3D structure. An alternate 3D printing technique utilizes liquid metal to additively manufacture metal objects. This process, generally referred to as "material jetting" is analogous to inkjet printing [7] and can be implemented in two distinct: continuous jet and drop-on-demand (DOD). In the continuous jet method, a liquid metal jet is formed and caused to break up via a perturbation stimulus into a continuous stream of welldefined droplets (volume and velocity) at a fixed distance from the nozzle. The droplets needed for fabrication are deposited on the build substrate, the other droplets are deflected. In contrast, in the drop-on-demand method, well-defined 
droplets are ejected from a nozzle and deposited on a build substrate as needed. The precision of the DOD jetting process enables a reduction in material waste.


Fig. 1: MagnetoJet process overview: (a) cross-sectional view of printhead and other essential components (b) simulation model showing the magnetic field generated by a pulsed magnetic coil as well as the volume fraction of ejected liquid aluminum. Objects shown are not to scale.

In this paper, we provide an overview of a novel DOD metal AM technique based on magnetohydrodynamic droplet ejection that can be used to create 3D structures with complex geometries. We also demonstrate two computational models that are used to predict system performance and explore critical performance parameters. In this DOD process, a spooled solid metal wire, approximately $1 \mathrm{~mm}$ in diameter, is continuously fed to a ceramic reservoir of the nozzle where it is resistively heated to form $3 \mathrm{~mL}$ of molten aluminum (Fig. 1a). The molten metal flows from the reservoir into a nozzle via capillary forces. The nozzle is surrounded by a copper coil that is electrically pulsed to produce a transient magnetic field $\mathbf{B}$ within it. The magnetic field, in turn, induces a circulating current density $\mathbf{J}$, that back couples to the transient magnetic field thereby generating a magnetohyrodynamic Lorentz force density ( $\mathbf{f}_{\text {MHD }}$ ) within the ejection chamber, whose radial component creates a transient "effective pressure" $(\mathrm{P})$ pulse that ejects a liquid metal droplet through the orifice. The metal droplet travels through an argon shroud, which envelops the reservoir and the orifice, and is deposited on a stainless steel substrate that is heated to a temperature below the melting point of the metal. The droplets coalesce and solidify on the substrate to produce extended solid structures through layer-by-layer deposition, which is achieved by moving the substrate using computer numerical control (CNC) and computer aided design (CAD) files. Vader Systems have developed and commercialized this technology under the tradename MagnetoJet ${ }^{\mathrm{TM}}$.

The Magnetojet ${ }^{\mathrm{TM}}$ printing process has been used to create aluminum parts with a repetition rate up to 1000 droplets $/ \mathrm{sec}$, with a droplet placement resolution of $500 \mu \mathrm{m}$. It has achieved a mass deposition rate of up to $1 \mathrm{lb}$ per hour based on a single orifice that generate droplets with a $500 \mu \mathrm{m}$ diameter. In addition, it is a low cost process that can print parts with improved mechanical properties owing to the presence of unique metal grain structure [8]. In this work, we describe the fundamental principles of Magnetojet ${ }^{\mathrm{TM}}$ and introduce computational models that predict droplet deposition, coalescence and solidification and can be used to optimize the process. Sample 3D structures printed by Magnetojet ${ }^{\mathrm{TM}}$ are also demonstrated in this presentation.

\section{Process Description}

Vader Systems have developed and commercialized a prototype printing system with a printhead consisting of a 2part refractory nozzle, a solenoid coil which is water cooled and an argon gas shroud. Liquefaction of Al wire takes place at $1146-1246 \mathrm{~K}\left(850-950^{\circ} \mathrm{C}\right)$ by resistively heating the refractory reservoir. As new metal wire enters the reservoir, it is melted by thermal conduction via the already molten metal and the refractory nozzle. The lower part of the nozzle contains an orifice, ranging from 100-500 $\mu \mathrm{m}$ in diameter, through which liquid metal droplets are ejected. The size of droplets varies from 50-550 $\mu \mathrm{m}$ in diameter depending on orifice geometry, diameter, ejection frequency and pulse duration. Steady droplet ejection rates ranging from $40-1000 \mathrm{~Hz}$ with short burst ups up to $5000 \mathrm{~Hz}$ have been achieved with the machine 
prototype. Common Aluminum alloys such as 4043, 6061 and 7075 have been used to successfully print solid metal structures.

\section{Computational Models}

Computational simulations were performed prior to prototype fabrication and during the design cycle to optimize selected process parameters for performance, i.e. pressure buildup near the orifice, droplet generation and ejection, dropletair interaction and droplet-substrate interaction. We performed computational fluid dynamics (CFD) analysis using the multiphysics $\boldsymbol{F L O W - 3 D}$ (www.flow3d.com) software to study the magnetohydrodynamic aspects of droplet generation and the thermo-fluidic aspects of droplet deposition, coalescence and solidification. In order to simplify the analysis, two complementary, but independent models were designed: one for droplet generation and another for droplet coalescence and solidification on the substrate surface. This approach allows for analysis of the critical control parameters that affect each phenomenon independently, thus decoupling a process that would be too difficult to model with all relevant electromagnetic and thermofluidic physics included. Droplet generation is affected by the coil pulse duration, voltage and
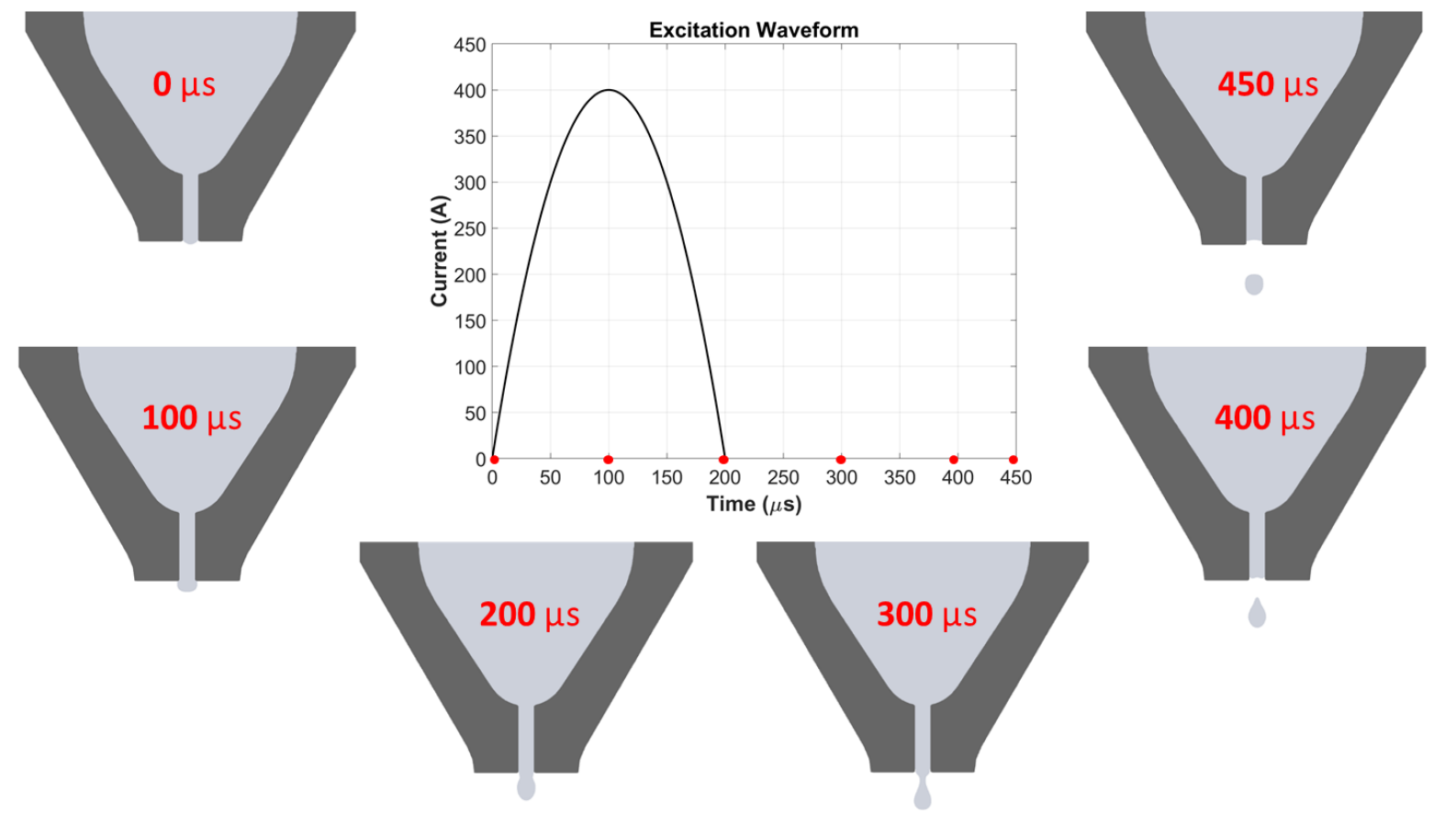

Fig. 2: Example of a current pulse applied to the ejection coil. Droplet generation is plotted as $2 \mathrm{D}$ slices of the fluid at the $300 \mu \mathrm{m}$ ejection orifice as a function of time.

current amplitudes, wave shape, molten metal conductivity and the relative geometries of the coil and the molten metal. Solidification of droplets on the substrate is influenced by various factors such as droplet ejection frequency, temperature, velocity, size, center-to-center droplet spacing, substrate and surrounding temperature and others.

In order to fabricate precise 3D metal solid structures, droplet patterning, coalescence and solidification are critical. In this process, droplets are ejected with a velocity ranging from 1-10 m/s and travel through an argon-shielded atmosphere before reaching the substrate. Thermal diffusion from droplet to surrounding atmosphere takes place during flight and after impacting the substrate surface, droplet solidification takes place owing to the thermal diffusion from droplet to substrate. The substrate is heated to a temperature that is below the melting point of the metal. This reduces the temperature gradient between droplet and substrate and hence, slows down the rate of thermal diffusion. This promotes smoother coalescence with the neighboring droplets (interlayer) and between layers (interlayer) as well the growth of favorable metal microstructure, thus creating 3D solid structures with low porosity and no undesired layering artifacts. 


\subsection{Droplet Generation}

In the first model, FORTRAN 90 code was used to calculate the Lorentz force density around an axisymmetric solenoid as a function of time, spatial conductivity, number of coil turns and the applied current amplitude and wave shape. This code was used in customizing the commercially available $\boldsymbol{F} \boldsymbol{L} \boldsymbol{O} \boldsymbol{W}-3 \boldsymbol{D}$ program by including the Lorentz force density as a force acting on individual fluid elements. This CFD analysis was used to study MHD-based droplet ejection behavior and effective pressure generation. The computational domain (CD) is axisymmetric with respect to the centerline of the ejection nozzle, and so it was constructed in cylindrical coordinates. The most significant component of the MHD model is the magnetohydrodynamic force $f_{M H D}$, which causes the motion of the metal in the reservoir and through the ejection orifice. A time dependent eddy current computation, based on analytical equations of the magnetic vector potential $\mathbf{A}$ and magnetic flux density $\mathbf{B}$ around an axisymmetric solenoid was performed to calculate this force density. The analytical equations, derived by Callaghan and Maslen in [9], were adapted to the CFD model and used to calculate the Lorentz-like MHD force via equation set (1).

$$
\begin{gathered}
\boldsymbol{B}=\nabla \times \boldsymbol{A} \\
\boldsymbol{E}=-\frac{\partial \boldsymbol{A}}{\partial t} \\
\boldsymbol{J}=\sigma \boldsymbol{E} \\
f_{M H D}=\boldsymbol{J} \times \boldsymbol{B}
\end{gathered}
$$

Since both the circulating electric field and the magnetic flux density can be thought of as originating from a single magnetic vector potential A, both of these fields are directly obtained from the analytical solution for A. To obtain the circulating current density $\mathrm{J}$, electric field is multiplied by the conductivity of liquid aluminum at $1023 \mathrm{~K}$, obtained from [10]. The magnetic vector potential, magnetic flux density, induced current density J, and the MHD force values were validated via comparison to those calculated by the finite element software COMSOL (www.comsol.com) for accuracy.

Figure 2 shows an example of a current excitation pulse applied to the ejection coil, along with 2D temporal slices of aluminum droplet ejection from a $300 \mu \mathrm{m}$ diameter orifice. The velocity of the droplet was predicted to be $2 \mathrm{~m} / \mathrm{s}$, which agrees with the average experimental velocity of $2.5 \mathrm{~m} / \mathrm{s}$. It is important to note that the slope of this excitation current corresponds to the generated pressure, i.e. a positive current slope will generate a downward pressure and a negative slope will cause a retraction pressure. Both of these pressures, corresponding to the direction of $f_{M H D}$, are necessary to push liquid out of the orifice and then immediately pull it back to ensure a droplet pinch-off. Since the effective time for the droplet generation and droplet pinch-off rate are in the order of $400 \mu \mathrm{s}$, the current pulse is half of this time, allowing for a DOD ejection of liquid metal. Since the droplet ejection is in an on-demand mode, it is reasonable to use the data for droplet size and velocity from the first model as input into the droplet deposition model, which is discussed next.

\subsection{Droplet Deposition}

In the droplet deposition model, we designed CFD models to investigate the droplet deposition, coalescence and solidification on a heated substrate. A Finite Volume thermo-fluidic analysis was performed using the solidification model in $\boldsymbol{F L O W - 3 D}$. The temperature-dependent physical properties of molten aluminum, such as viscosity, density, heat capacity and thermal conductivity were obtained from [11-14]. Morphology of inclined pillars were studied as a function of droplet overlap fraction. In this analysis, spherical droplets of molten aluminum at $1023 \mathrm{~K}$ impact a stainless steel substrate, kept at $473 \mathrm{~K}$, from a height of $3 \mathrm{~mm}$. The droplets have a diameter of $450 \mu \mathrm{m}$ and travel with an initial velocity of $2.5 \mathrm{~m} / \mathrm{s}$. Various droplet ejection frequencies were studied and it was observed that at frequencies above $100 \mathrm{~Hz}$, the temperature of the solidified droplet is too high such that when the next droplet impacts it, excessive re-melting takes place and the structure is not stable. This effect was also observed during experiments, with the frequency limited to $20 \mathrm{~Hz}$ to ensure full coalescence and solidification of each droplet. When building pillar structures using the DOD process, it is critical to control the droplet overlap fraction, which is defined as the ratio of the maximum overlap length between any two droplets to the outside diameter either droplet (Fig. 3e). This variable is controlled by the relative velocities of the droplet and the moving substrate during experiments, and via $\mathrm{x}$-coordinate placement of the droplet in the model. Structures of 10 droplets at an ejection frequency of $100 \mathrm{~Hz}$ are presented in Fig. 3, with overlap fractions of $0.50,0.80$ and 
1.00 (vertical pillar). In Figure 3a, the overlap fraction is assumed to be 0.5 and the result is a flat coalesced layer. In Fig. $\mathbf{3 b}$, the overlap fraction is 0.8 which leads to formation of an inclined structure at $45^{\circ}$ whereas in Fig. 3c the overlap fraction is 1.0 and as expected, the result is a vertical pillar. Pillars with a range of inclination angles can be fabricated by varying the overlap fraction during deposition. This is shown in Figure 3(d), which is a snapshot of the experimentally printed structures.

\section{Conclusion}

We have presented an overview of a MHD-based DOD metal process for printing metallic 3D structures. The MagnetoJet ${ }^{\mathrm{TM}}$ process is able to manufacture parts via a layer-by layer approach using aluminum alloys such as 4043,6061
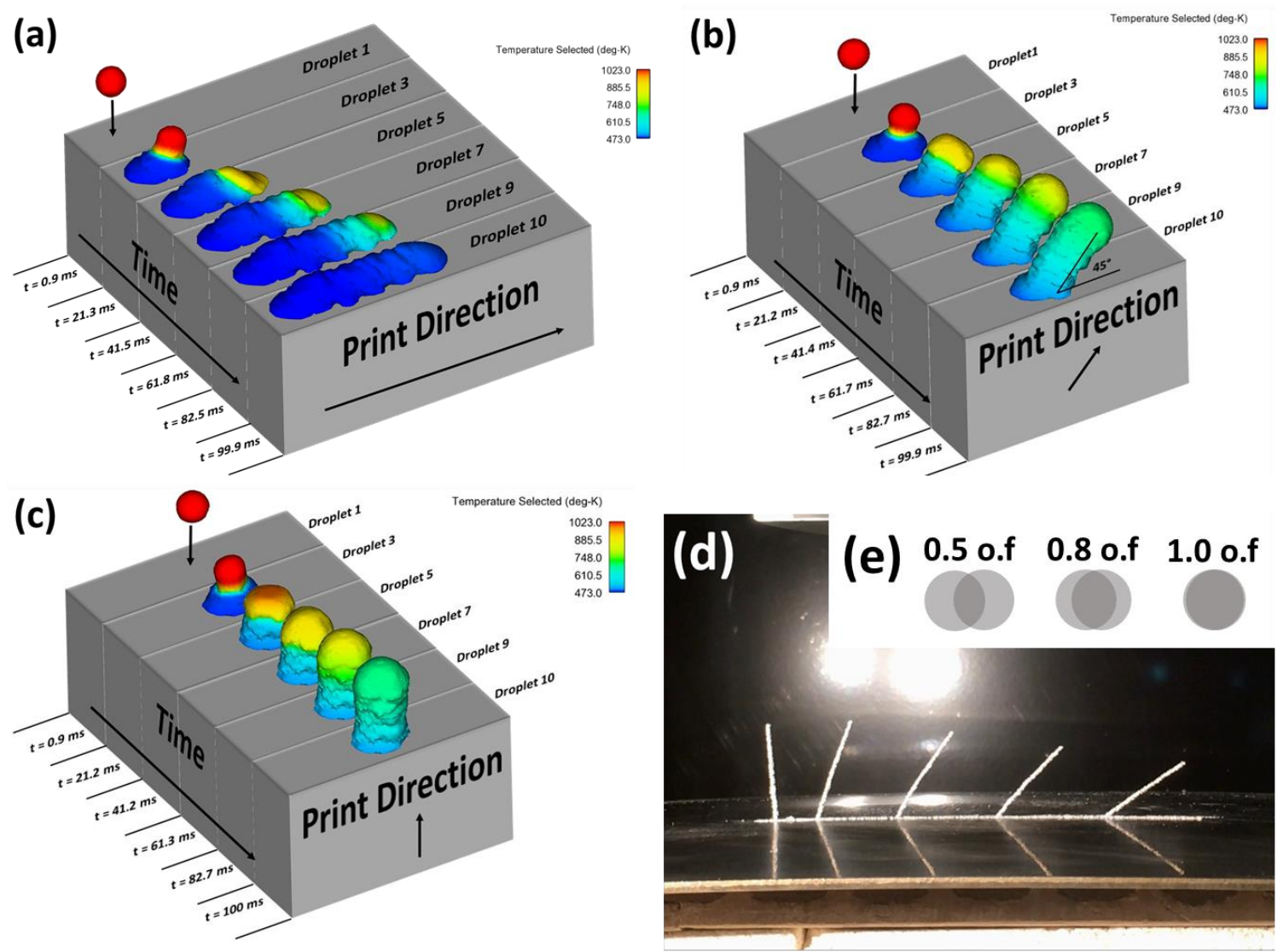

Fig. 3: The effect of droplet overlap fraction on the DOD MagnetoJet process: (a) 10 droplets at $100 \mathrm{~Hz}, 0.5$ overlap fraction; (b) 10 droplets at $100 \mathrm{~Hz}, 0.8$ overlap fraction with a $45^{\circ}$ incline; (c) 10 droplets at $100 \mathrm{~Hz}, 1.0$ overlap fraction; (d) experimentally printed pillars; (e) Depiction of the overlap fraction via a top-down view of two deposited droplets (image in (d) is courtesy of Rochester Institute of Technology, all rights reserved).

and 7075. Droplets of sub-milimeter size are ejected from the printhead by MHD force up to kHz frequencies. Deposition rates up to $1 \mathrm{lb}$ per hour has been achieved based on 500 micron droplet size. The process is extremely cost efficient since it uses metal wire feedstock, thus eliminating the need of specially prepared powders. Still, challenges remain in realizing the optimum operating parameters of operation, and improvement of overall process performance in terms of capacity, thermal efficiency, metallurgical bonding and resolution is needed. Specifically, identifying crucial droplet generation parameters such as orifice size, ejection nozzle geometry and parameters of the actuation waveform will allow for more precise and faster droplet deposition. Additionally, isolating the critical droplet deposition parameters will allow the process to build a broader range of metallic structures, such as inclined pillars, horizontal overhangs, etc. To address this, we have presented a computational modelling approach, composed of two complementary models: a droplet generation 
model and a droplet deposition model. These models can be used for a rational study and design of the MagnetoJet process, as well as similar DOD processes and will be improved in the future to include additional physics, in order to faithfully model the process in its entirety.

\section{References}

[1] J. Jang and S. S. Lee, "Theoretical and experimental study of MHD (magnetohydrodynamic) micropump," Sensors \& Actuators: A. Physical, vol. 80, no. 1, pp. 84-89, 2000.

[2] D. S. Thomas and S. W. Gilbert, "Costs and cost effectiveness of additive manufacturing: A literature review and discussion," in NIST Special Publication, p. 1176, 2015.

[3] P. Li, S. Q Ji, X. Y. Zeng, Q. W. Hu, W. H. Xiong, "Direct laser fabrication of thin- walleded metal parts under open-loop control," International Journal of Machine Tools \& Manufacture, vol. 47, 2007.

[4] I. Yadroitsev, I. Shishkovsky, P. Bertrand, I.Smurov, "Manufacturing of fine-structured 3D porous filter elements by selective laser melting," Applied Surface Science, vol. 255, 2009.

[5] H. Tan, J. Chen, F. Y. Zhang, X. Lin, W. D. Huang, "Process analysis for laser solid forming of thin-walled structure," International Journal of Machine Tools \& Manufacture, vol. 50, 2010.

[6] X. Li, C. Wang, W. Zhang, Y. Li, "Fabrication and characterization of porous Ti6Al4V parts for biomedical applications using electron beam melting process," Materials Letters, vol. 63, pp. 403-405, 2009.

[7] M. Suter, E. Weingärtner, and K. Wegener, "MHD printhead for additive manufacturing of metals," Procedia CIRP 2, pp. 102-106, 2012.

[8] M. Orme and R. F. Smith, "Enhanced aluminum properties by means of precise droplet deposition," Journal of Manufacturing Science and Engineering, Transactions of the ASME, vol. 122, no. 3, pp. 484-493, 2000.

[9] E. E. Callaghan, S. H. Maslen and Lewis Research Center, "The magnetic field of a finite solenoid," National Aeronautics and Space Administration, 1960.

[10] P. D. Desai, H. M. James and C. Y. Ho, J. Phys. Ref. Data, vol 13, no. 4, p. 1131, 1984.

[11] L. Battezzati and A. L. Greer, "The viscosity of liquid metals and alloys," Acta Metallurgica, vol. 37, no. 7, pp. 1791-1802, 1989.

[12] W. D. Drotning, High Temperature Science, vol. 11, p. 265, 1979.

[13] B. J. McBride, J. Gordon and M. A. Reno, "Thermodynamic data for fifty reference elements," National Aeronautics and Space Administration, Office of Management, Scientific and Technical Information Program. Springfield, Va; Washington, DC, 1993.

[14] C. Y. Ho, R. W. Powell and P. E. Liley, "Thermal Conductivity of the Elements," Journal of Physical and Chemical Reference Data, vol. 1, no. 2, pp. 279-421, 1972. 